\section{Pacific Northwest National Laboratory \\ Operated by Battelle for the} U.S. Department of Energy

\title{
A Fiber-Optic Neutron Detector for a Drive-By Scenario
}

\author{
Dr. Harry S. Miley
}

February 1999

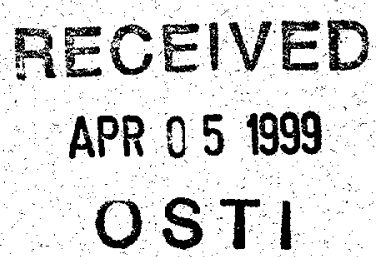

Prepared for the U.S. Department of Energy under Contract DE-AC06-76RLO 1830 


\section{DISCLAIMER}

Portions of this document may be illegible in electronic image products. Images are produced from the best available original document. 


\title{
A Fiber-Optic Neutron Detector for a Drive-By Scenario \\ Dr. Harry S. Miley
}

\author{
February 1999
}

Prepared for the U.S. Department of Energy

Under Contract DE-AC06-76RLO 1830

\section{Pacific Northwest National Laboratory}

Richland, Washington 


\section{Executive Summary}

The measurement scenario of a neutron source driving by a detector has been evaluated. It is possible to use PNNL lithium-loaded fiber optics to measure the source, even at reasonably high speeds. A detector sufficient to detect the neutrons from the source at a high confidence level (see below for computation method) can be produced in a compact and robust configuration for a reasonable cost. In addition, the PNNL solution measures gamma-ray signals and will effectively add the function of a proximity sensor, lower the false-alarm rate, and allow discrimination between certain neutron source scenarios. Finally, the need for definition of confidence levels (both the method of computation and the required false alarm probability), emplacement form-factor, and electronic interface is required of a potential user to revise or customize the design outlined in this paper.

\section{Introduction}

The Drive-By scenario has been one of concern for a number of decades and of importance to many application areas: Safeguards, Radiation Protection, Nuclear Smuggling, Border Control, and others. PNNL has developed sensors for these and other neutron measurement applications using proprietary fiber-optic detectors designed for low-profile, high-sensitivity measurements.

The measurement science of these efforts often revolves around plutonium due to its importance in nuclear weapons. The radiation signature of plutonium is highly variable depending on its nuclear and chemical history. Plutonium, specifically ${ }^{239} \mathrm{Pu}$, is made in reactors from the irradiation of uranium fuel. After a ${ }^{239} \mathrm{Pu}$ atom is created, it may be transmuted into ${ }^{240} \mathrm{Pu}$ by the (unwanted) absorption of another neutron. The proportion of ${ }^{240} \mathrm{Pu}$ in plutonium is therefor referred to as its 'burn-up'. Many tests of PNNL detectors have been performed with plutonium sources that have about $6 \%$ burn-up. This is important, since a large fraction of the neutrons emitted by a source come from the ${ }^{240} \mathrm{Pu}$. (Please refer to Appendix I for a typical plutonium component analysis.) However, the matrix of the plutonium source is of extreme importance, as well. Plutonium metal emits neutrons from spontaneous fission only, while certain $(\alpha, n)$ reactions on oxygen cause more neutrons to be emitted. Finally, $(\alpha, n)$ reactions on fluoride increase in the number of neutrons emitted about 20 fold. It should be noted that comparisons made below include both oxide and bare metal sources.

A PNNL lithium-loaded fiber optic detector works by scintillation. Ionizing radiation causes electrons to be stripped from atoms, and some of the recombination energy is given off in the form of light, which is captured by a photo-multiplier tube (PMT), amplified, and counted. Neutrons are only mildly ionizing, so the neutron sensitivity of the fiber is dramatically increased by adding $6 \mathrm{Li}$, which has a 1360-barn cross section for neutrons. Upon absorbing a neutron, the $6 \mathrm{Li}$ atom essentially explodes into $4 \mathrm{He}$ and $3 \mathrm{H}$ nuclei, which are highly ionizing and therefore usually deposit a large amount of energy all in one fiber. By comparison, a gamma-ray interacts via the photo-electric, Compton, or pair-production processes and thereby gives all its energy to an electron. Electrons deposit little energy in a single fiber and usually penetrate about four fibers.

Adding lithium to the fibers dramatically enhances neutron detection capability over gamma-ray detection capability. However, gamma-ray sensitivity is desirable, as long as the two types of signals can be discriminated. Various discrimination techniques for the fiber have been invented at PNNL, including simple pulse-height discrimination, double-ended coincidence requirements (a PMT at each end of the fiber), position sensitivity (single-fiber neutrons vs. multi-fiber gamma-rays), and a high-speed, pulse counting technique. These different methods lend themselves more or less to specific application requirements. Optimum results are usually obtained by making a custom combination of electronic techniques. For instance, neutron and gamma-ray signals can be combined to increase detection sensitivity in certain applications. 


\section{Sensitivity}

The sensitivity of a counting system can be defined in several ways. It is important, when designing a multi-element system, to specify sensitivities in a consistent and correct manner. While Currie describes a rigorous method for defining a minimum detectable activity (see Appendix 2), in this paper we will simply compare estimated signal levels to measured or inferred background levels to estimate the detection. For this reason, a figure of merit for a measurement scenario can be constructed based on the following parameters:

$\begin{array}{ll}\text { Specific Activity: } & \text { neutrons per second per gram } \\ \text { Source Mass: } & \text { grams } \\ \text { Source Distance: } & \text { meters } \\ \text { Measurement Interval/Time: } & \text { seconds } \\ \text { Detector Area: } & \text { square meters } \\ \text { Detector Efficiency: } & \text { counts/incident neutron } \\ \text { Background: } & \text { counts per measurement interval }\end{array}$

First, the incident number of neutrons should be computed:

$$
\text { Incident Neutrons } \left.=(\text { Specific Activity }) *(\text { Mass }) *(\text { Time }) /\left(4 \pi\left(\text { Distance }^{2}\right)\right) *(\text { Area })\right)
$$

Then the sensitivity may be estimated as:

$$
\text { Sensitivity }(\sigma)=(\text { Incident Neutrons }) *(\text { Efficiency }) /(\text { Background })^{1 / 2}
$$

Given these definitions, it is possible to compare several recently measured detector-source scenarios, along with certain hypothetical scenarios (Table 1). The 'drive-by' scenario assumes $6000 \mathrm{~g}$ of $2.5 \%$ burnup material moving at $20 \mathrm{~m} / \mathrm{s}$ at $4 \mathrm{~m}$ closest approach to the detector. Inferred data is italicized.

Table 1. Sensitivity Comparison (See Figure 1 for further explanation).

\begin{tabular}{lcccc}
\hline Detector & Proposed & Tower & Asphalt & Asphalt \\
\hline Location & drive-by & 3745 & 3745 & IT\&T \\
& & & & \\
Matrix & metal & oxide & oxide & metal \\
Burnup \% & 2.5 & 6 & 6 & 6 \\
n/s/g & 26.18 & 114.64 & 114.64 & 62.84 \\
Mass $(g)$ & 6000 & 98 & 98 & 20 \\
Activity & 157097 & 11235 & 11235 & 1257 \\
\hline & & & & \\
Distance $(m)$ & 4 & 1 & 1 & 1 \\
Time $(s)$ & 0.40 & 10 & 10 & 1 \\
Det area $\left(m^{2}\right)$ & 0.21 & 0.42 & 0.43 & 0.43 \\
Incident Neutrons & 65.63 & 3725.22 & 3860.52 & 43.18 \\
\hline & & & & \\
Source Counts & 19.43 & 1378.33 & 1302.93 & 14.68 \\
Background $(\mathrm{n})$ & 3.08 & 370 & 399 & 7.70 \\
\hline Sensitivity $(\sigma)$ & 11.07 & 71.66 & 65.23 & 5.29 \\
\hline
\end{tabular}

The sigma level of the proposed drive by measurement does not tell the complete story, since it was derived using simple square-root estimation. In stead, the overlap of probability distributions should be examined. Probability distributions (poissonian) for a background rate of 3.08 counts per interval and a signal rate of 
19.80 counts per interval are shown in Figure 1. A critical level, $\mathrm{L}_{\mathrm{c}}$, can be established at about 9 counts such that $0.5 \%$ of the Background probability is above the limit and $0.5 \%$ of the Signal probability is below the limit. In this way, it can be seen that the false positive and false negative probabilities are $0.5 \%$. Given that a $99.75 \%$ confidence limit is usually called " 3 -sigma" confidence, we can say that the signal anf background here are separated by a little less that 6 sigma. The drive-by scenario $(20 \mathrm{~m} / \mathrm{s}$ and $4 \mathrm{~m})$ would then have Minimum Detectable Activity, $L_{d}$ of $6 \mathrm{~kg} 2.5 \% \mathrm{Pu}$ at $0.5 \%$ false positive/negative probability.

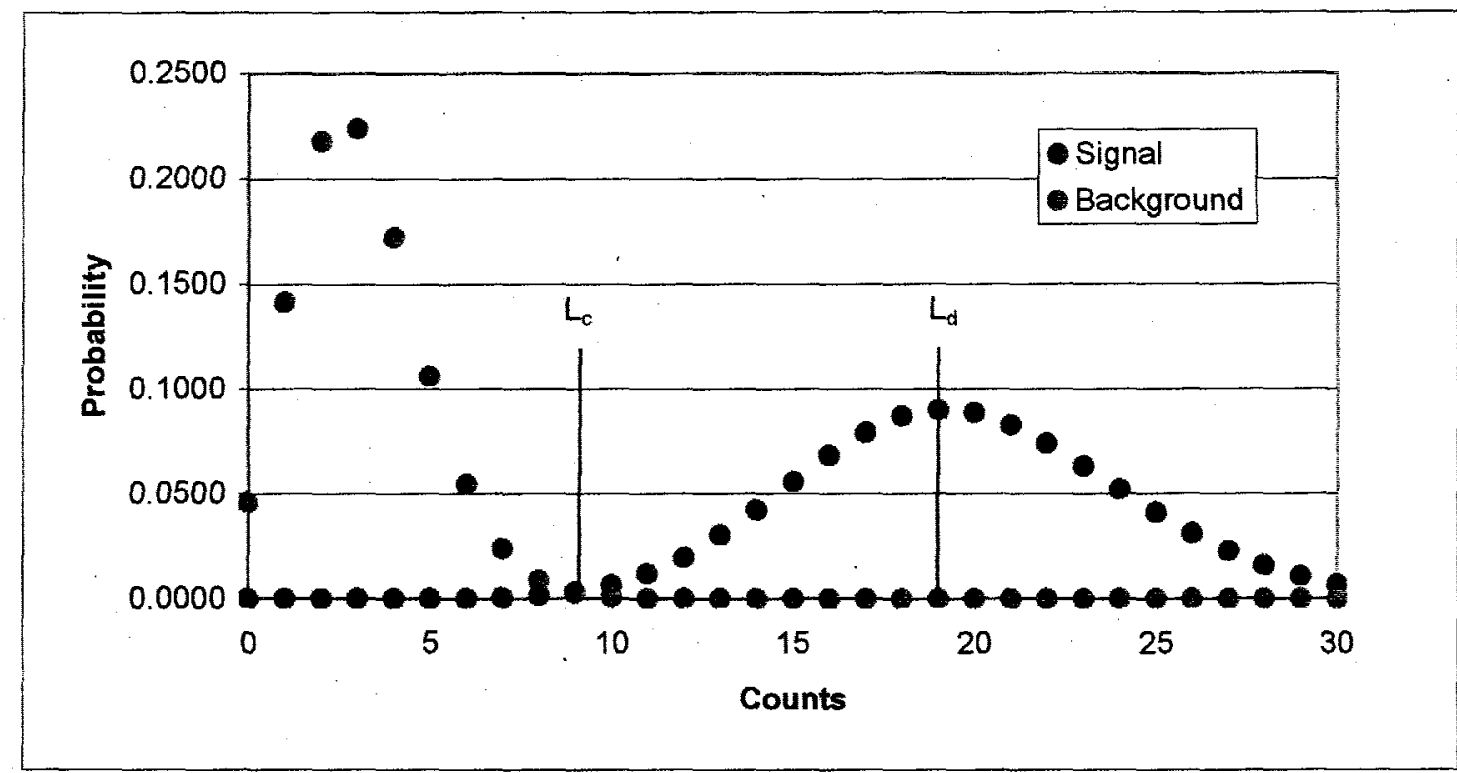

Figure 1. Probability Distributions

A false positive rate of $0.5 \%$ is not as good as it sounds at first reading. Given a measurement period of 0.4 seconds and 86400 seconds per day, there should be over 1000 false positives per day (i.e. measurements above $L_{c}$ ). A proximity sensor can reduce this drastically. It should be noted that since the fiber optic electronics package returns gamma-ray as well as neutron data, that the gamma-ray data may be used to increase the effective sensitivity of the detector package. Outdoor gamma-ray background is by its nature variable, so that a Poisson distribution of the average gamma-ray background may provide too low an estimator of fluctuations. Nevertheless, the existence of a gamma-ray peak in coincidence with a neutron peak of low significance greatly bolsters the confidence level that a plutonium source signal has been detected.

\section{Drive-By Scenario}

The Drive-By scenario, shown schematically in Figure 2, can be theoretically modeled using a few simple parameters: the source strength, $A$, the source velocity, $v$, the distance of closest approach, $b$, and the detector background, $B$.

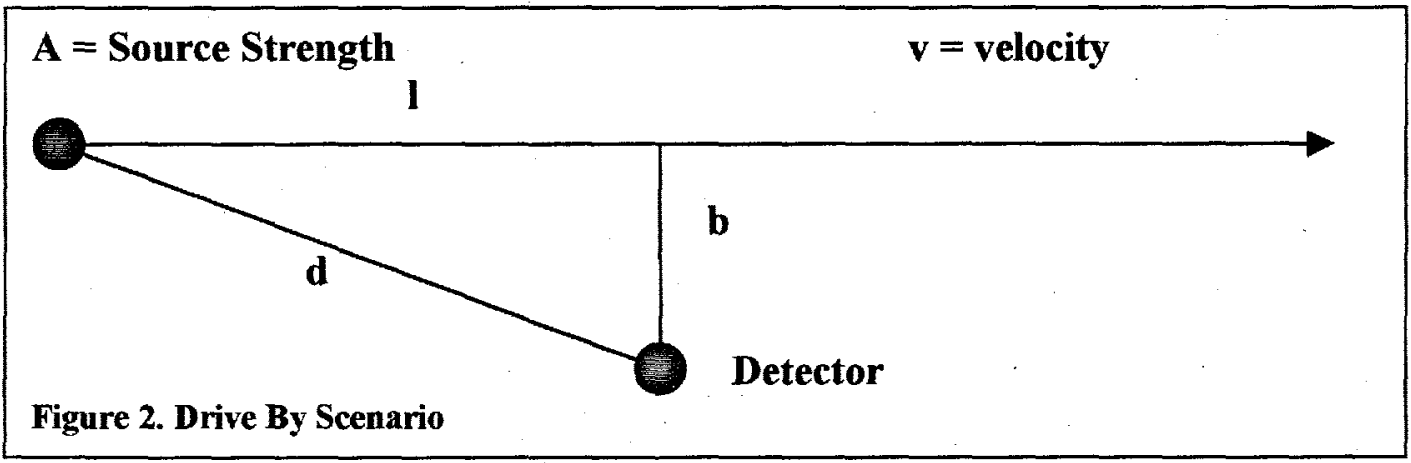


If detector efficiency is given as $\varepsilon$, we can create a simple equation to describe the signal as a function of time. This simple model ignores the orientation of the detector, and takes no complex moderation effects into account.

$$
\begin{aligned}
& S(t)=B+\frac{A \varepsilon}{d^{2}}=B+\frac{A \varepsilon}{l^{2}+b^{2}}=B+\frac{A \varepsilon}{b^{2}+\left(\left(t-t_{0}\right) v\right)^{2}} \\
& S(t)=B+\frac{A \varepsilon}{b^{2}+\left(v t-x_{o}\right)^{2}}
\end{aligned}
$$

An example of the success of this simple model is given in Figure 2. The fitting program was allowed to choose any values for $\mathrm{A}, \mathrm{b}$ and $\mathrm{v}$. Unfortunately these parameters are correlated such that if one wishes to

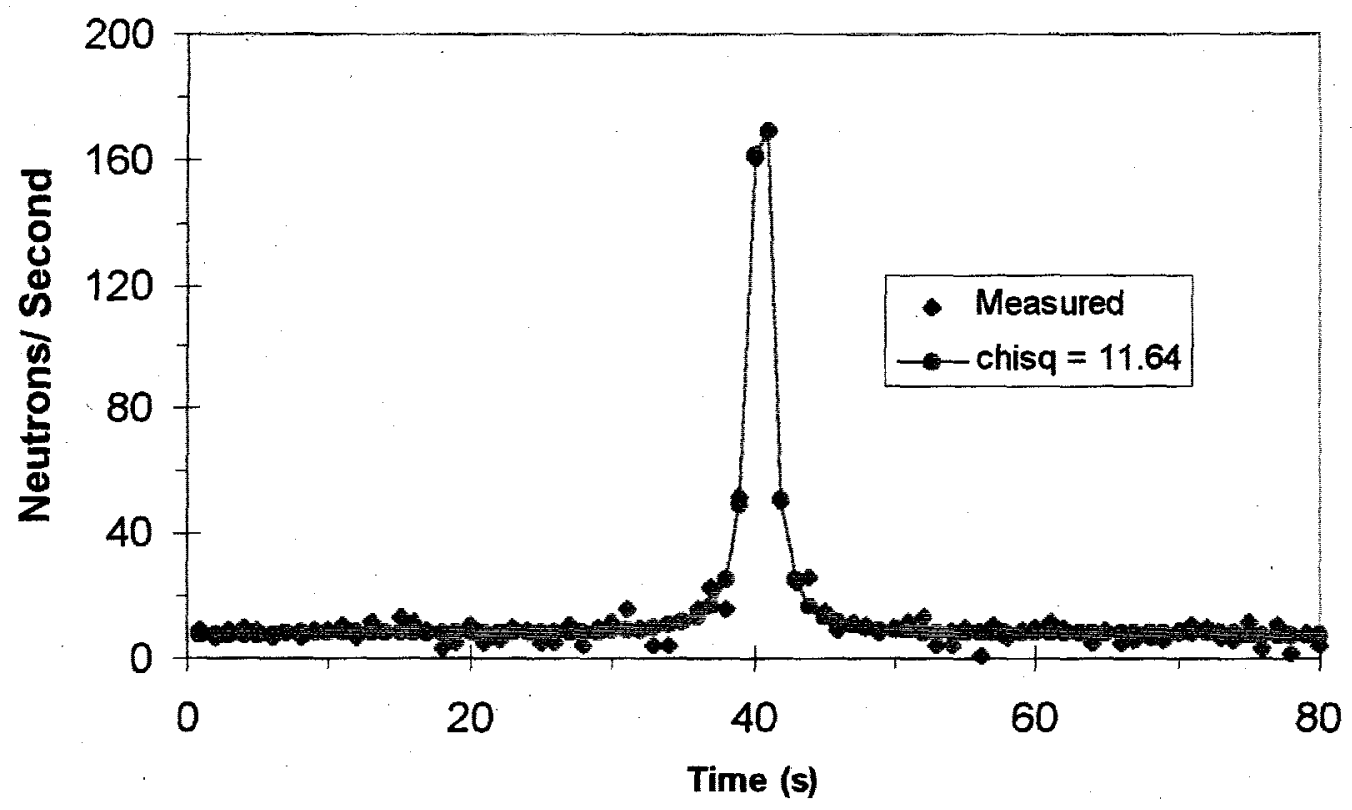

Figure 3. Actual drive-by data taken with Asphalt detector.

constrain $A$, suitable values for $b$ and $v$ must be known. It is possible to fix any value desired for any one variable ( $A, b$, or $v$ ) and adjust the other two to obtain an excellent fit of the simple model to the data. In the case of Figure 3, a large source was driven at about $2 \mathrm{~m} / \mathrm{s}$ past an Asphalt sensor. The distance of closest approach of the source was about $2 \mathrm{~m}$. The individual neutron measurements were taken on 1 second time intervals. Knowing these values for $v$ and $b$, it is possible to reasonably estimate $A$, the rate of neutron emissions from the source.

It should be noted that the additional information provided by distance of closest approach, $b$, and velocity, $v$, also allow the discrimination of a point source from a distributed source by comparing the model and observed width of the peak. This is because the width of the peak, for instance at the half-maximum level, is a simple function of $b$ and $v($ e.g. FWHM $=2 b / v)$. This could allow the discrimination of a shipment of hazardous waste from a concentrated source, and is equally true of the gamma-ray signals as the neutron signals, although in both cases scattering will cause this approach to be somewhat limited in usefulness.

The additional signal provided by the gamma-ray channel of the lithium-loaded fiber-optic detector allows simple discrimination of spoofing sources from a variety of source types. To be specific, for the IT\&T Demo, it was possible to 'train' the alarm algorithm of the Asphalt detector such that certain neutron-to- 
gamma-ray ratios would clearly discriminate between a gamma-ray source from a neutron source. In addition the two neutron sources could be identified by their ratio. However, subsequent testing (IT\&T Demo, 6 October, 1994) showed that the neutron-to-gamma-ray-ratios to be too variable to reliably differentiate between the neutron sources. This may have been due to differing amounts of gasoline (a neutron scatterer) in the vehicle tank or variation of some other uncontrolled parameter.

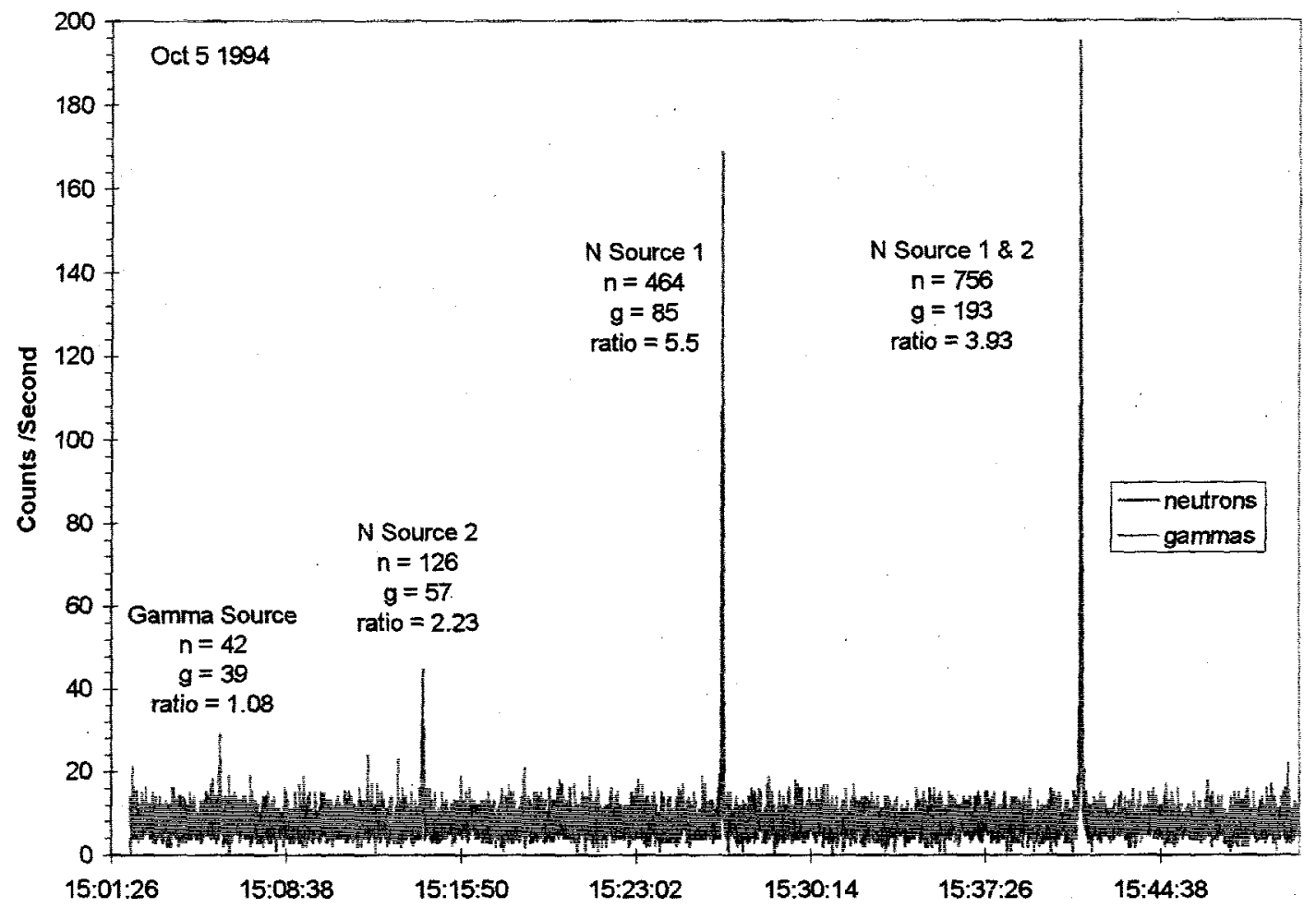

Figure 4. IT\&T Demo data

As a result of the success of the Asphalt sensor in IT\&T Demo, a variant on the Asphalt detector was produced. This detector and its electronics were customized for low power consumption, low-profile emplacement, low cost, and Lonworks ${ }^{\mathrm{TM}}$ network interoperability with a range and velocity sensor. As a result, the new sensor does not need a dedicated computer, simply a micro-controller which sums digital pulses (representing neutrons and gamma-rays) into 1 second intervals, then passes the interval data onto the network to be gathered by a central computer which displays and processes the multi-sensor data.

The implications of the new detector design are that the minimum power and cost per square meter of detector can be achieved as well as the highest ratio of active detector area to detector footprint. The physical packaging of the detector is in a multi-layer fabric enclosure with selectable stiffeners to give the unit varying degrees of flexibility. It can, for instance, be attached to a telephone pole, laid in wet grass, operated in a moving vehicle, including aircraft, or operated from a backpack or suitcase.

As an example of the results obtainable with the proposed detector, using the assumptions of Table 1 , and the simple theory put forth above, one would expect signals like those shown in Figure 5 . The background counts have been synthesized using a Poisson distribution about 3 counts per 0.4 second measurement interval. The signal, 19.8 counts, has been ratioed from experimental data (source activity, distance, velocity, and detector size). It should be noted that many other outcomes of the same parameters are possible due to normal statistical fluctuations. 


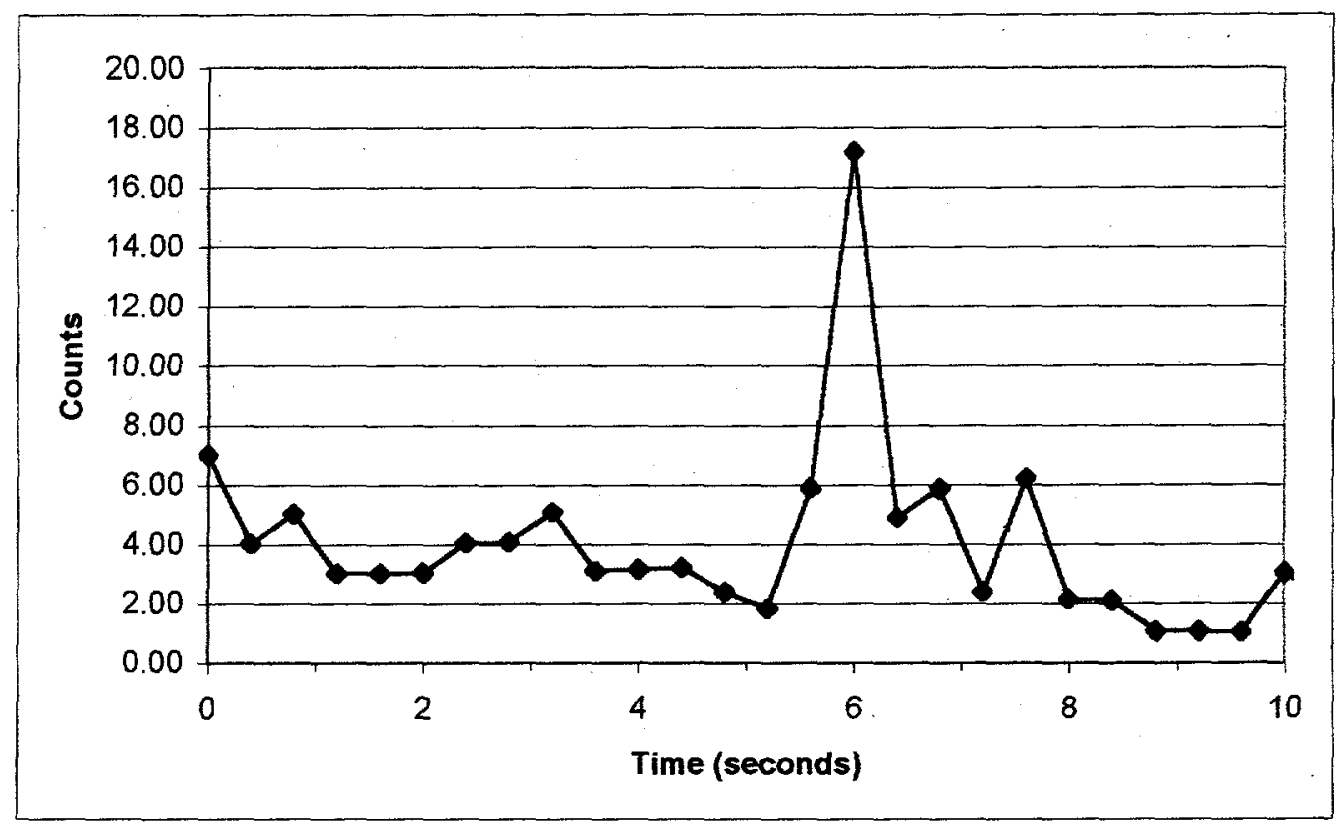

Figure 5. Simulated data from proposed detector

Another consideration in the design of a roadside detector is the physical layout of the detector. A detector of $0.21 \mathrm{~m}^{2}$ area would consist of about 14,500 meters of fiber and may be laid out as 14,500 fibers of $1 \mathrm{~m}$ length (Tower geometry) or 7750 fibers of $2 \mathrm{~m}$ length (Asphalt geometry). The PNNL fibers are wound onto a $2 \mathrm{~m}$ drum, thus integral fractions of $2 \mathrm{~m}$ length are cost effective. There are several tradeoffs in these choices. A long, thin detector (Asphalt geometry) will have a slightly reduced effective efficiency due to geometrical considerations at close ranges ( $13 \%$ less than Tower at 1 meter), but will be less sensitive to changes in source location along it's long axis. Also, the longer the fiber the less scintillation light that arrives at the PMT due to light loss in the glass. Thus, since neutron/gamma-ray identification depends on signal strength, shorter detectors have better neutron/gamma-ray separation.

Regardless of detector aspect ratio, sensitivities reported above have been measured in laboratory conditions with ample moderator (sheets of polyethylene) external to the detector. The sources were suspended at varying heights above the detector $(0-6 \mathrm{~m})$ with varying amounts of polyethylene above and below the detector, which was resting on a concrete floor. This is quite similar to a roadway scenario, if we assume that we can also add moderator to the roadway to achieve optimal neutron thermalization.

A long-term roadside detector may need special packaging. The Asphalt detectors are packaged in a relatively expensive welded aluminum box which protects the sensor from the elements, but does not necessarily support a heavy vehicle driving over. One obvious solution is to package the sensor in a relatively inexpensive plastic enclosure, then protect the sensor with a heavy piece of iron. This would allow a simple groove $20-40 \mathrm{~cm}$ wide to be cut perhaps $7 \mathrm{~cm}$ deep in an existing asphalt surface. Additional depth allows polyethylene to be installed if the roadway surface has poor neutron-moderation capabilities.

In addition to standard road-side or roadway installations, the sensor may be embedded into a concrete lane divider, but substantial thickness will be needed for neutron moderation ( 7 or more $\mathrm{cm}$ ). Other alternatives include overhead structures. However, while overhead structures may be simpler to erect, they likely cause the source to be more distant from the sensor. 


\section{Conclusions}

It is not possible to conclude that the proposed detector will perform in a satisfactory way in a drive-by scenario without first clearly defining the measurement standards needed. Specifically, false positive and negative probabilities must be defined for a particular application, while they have been assumed here to be $0.5 \%$. It is also not possible to adequately estimate costs without clearly defining the interface between the sensor package and emplacement parameters (size, weight, form factor, ruggedness, environmental conditions, etc). The flexibility of the fibers allows several emplacement options that compressed gas tubes do not, and could strongly affect total system cost. Finally, the electronic aspects of the detectors must dovetail with the overall system, which will affect the cost. Indeed, since the fiber-optic detector has the additional gamma-ray output perhaps even the system architecture could be improved.

The inclusion of gamma-ray signals could add a complete new dynamic to a drive-by system. Besides the obvious addition of bulk sensitivity, the possibility of added discrimination based on a simultaneous neutron/gamma-ray measurement has many possibilities.

PNNL has experimented with detection systems in drive-by scenarios for over 10 years. Systems have been deployed in demanding environmental conditions and in unusual platforms and performed very well. Previous projects have demanded that hundreds of thousands of meters of fiber be drawn in production conditions, thus all aspects of the process are neither research nor developmental. 